\title{
Modeling Clinical Tumors to Create Reference Data for Tumor Volume Measurement
}

\author{
Adele P. Peskin ${ }^{1}$ and Alden A. Dima ${ }^{2}$ \\ 1 NIST, Boulder, CO 80305 \\ 2 NIST, Gaithersburg, MD 20899
}

\begin{abstract}
Expanding on our previously developed method for inserting synthetic objects into clinical computed tomography (CT) data, we model a set of eight clinical tumors that span a range of geometries and locations within the lung. The goal is to create realistic but synthetic tumor data, with known volumes. The set of data we created can be used as ground truth data to compare volumetric methods, particularly for lung tumors attached to vascular material in the lung or attached to lung walls, where ambiguities for volume measurement occur. In the process of creating these data sets, we select a sample of often seen lung tumor shapes and locations in the lung, and show that for this sample a large fraction of the voxels representing tumors in the gridded data are partially filled voxels. This points out the need for volumetric methods that handle partial volumes accurately.
\end{abstract}

\section{Introduction}

The change in pulmonary nodules over time is an extremely important indicator of tumor malignancy and rate of growth. Physicians base both diagnoses and treatment on perceived changes in size, so accurate and precise measurement of such changes can have significant implications for the patient. Many articles have described a variety of techniques for calculating tumor volumes and/or the change in tumor size between two sets of data on the same tumor [1]-[6]. To compare volumetric techniques, we need reference data with a variety of known tumor volumes with different levels of background noise. Although phantom tumor data are currently available and published studies [7] [8] have compared volumetric methods on these phantom data sets, the phantom data settings are often not realistic, because the synthetic phantoms are placed in a synthetic background. A realistic and valid assessment of these volumetric methods needs realistic calibrated data sets. Lung tumor volume calculations are often difficult because of the physical location of tumors in the lung: typically highly vascular regions where tumors are hard to discriminate from overlaying blood vessels or along the pleural lining of the lung where boundaries are also hard to define. Ground truth sets of lung tumor data are needed to compare different volumetric measurement methods. Of particular importance are sets with a single tumor acquired over time. In a previous paper we presented a method for embedding spheres of known size into lung CT data to create reference synthetic 
data sets in realistic lung environments [9]. In this paper, we expand on these ideas to replicate eight sets of clinical data with a range of lung tumor shapes and locations. We explain why these sets were chosen, and then describe the methods to recreate clinical tumor shapes with synthetic tumors whose volumes are computed accurately as the shapes are created.

\section{Examination of Lung Tumor Edges}

The pixel intensities associated with a lung tumor appearing on a CT scan typically lie in the same intensity range as the blood vessels of the lung (between -100 and +200 Hounsfield units). The non-vascular lung tissue and air passageways, which serves as the background in these scans, appear at significantly lower intensities (-1000 to -700 Hounsfield unit range). The regions at or near the lung tumor surface lie in a broad range between the two (-700 to -100 Hounsfield units), and are only partially filled by a tumor or contain both tumor and other vascular material. Figure 1 shows a section of a CT slice through a tumor, along with a 3-D picture of an isosurface from 41 slices of the same data set that include the tumor. The isosurface is created at a Hounsfield value of -400 , and includes both the tumor and the surrounding blood vessels. A histogram of the pixel intensities in a small region containing the tumor, also shown in Figure 1 , is representative of diseased lung tissue. There is a peak for the background tissue, a peak for the tumor, and a spread of intensities in between the two that represents the partial volumes and vascular material surrounding the tumor.

\section{Clinical Tumor Sets}

Eight clinical tumors were selected with the help of a radiologist from a larger set of available data that included a range of sizes and physical locations in the lung. Images were obtained from the public NCI Reference Image Database to Evaluate Response (RIDER) [10]. Data from each of these patients are available at two different time points. We cannot precisely measure the volumes of these clinical tumors and their changes over time, but we can create synthetic models of known volume. Several of the selected tumors are small and centrally located in the lung. Others are larger and attached in some way to the lining of the lung. The larger tumors appear to be less round than the smaller ones, and require more complex geometric models. These eight tumors were selected because they had shapes and attachments representative of those seen in our data sets. Several of the tumors were selected because they represented lesions with commonly occurring challenges in terms of reading the data. If several sets of CT data included similar tumors, we chose a sample representative of that shape and location.

We are able to recreate clinical tumor shapes using combinations of spheres, ellipsoids, cones, and cylinders, which can be assembled at arbitrary sizes and angles with respect to one another. During assembly, partial voxel volumes are calculated by subdividing each voxel and testing for the appearance of each 

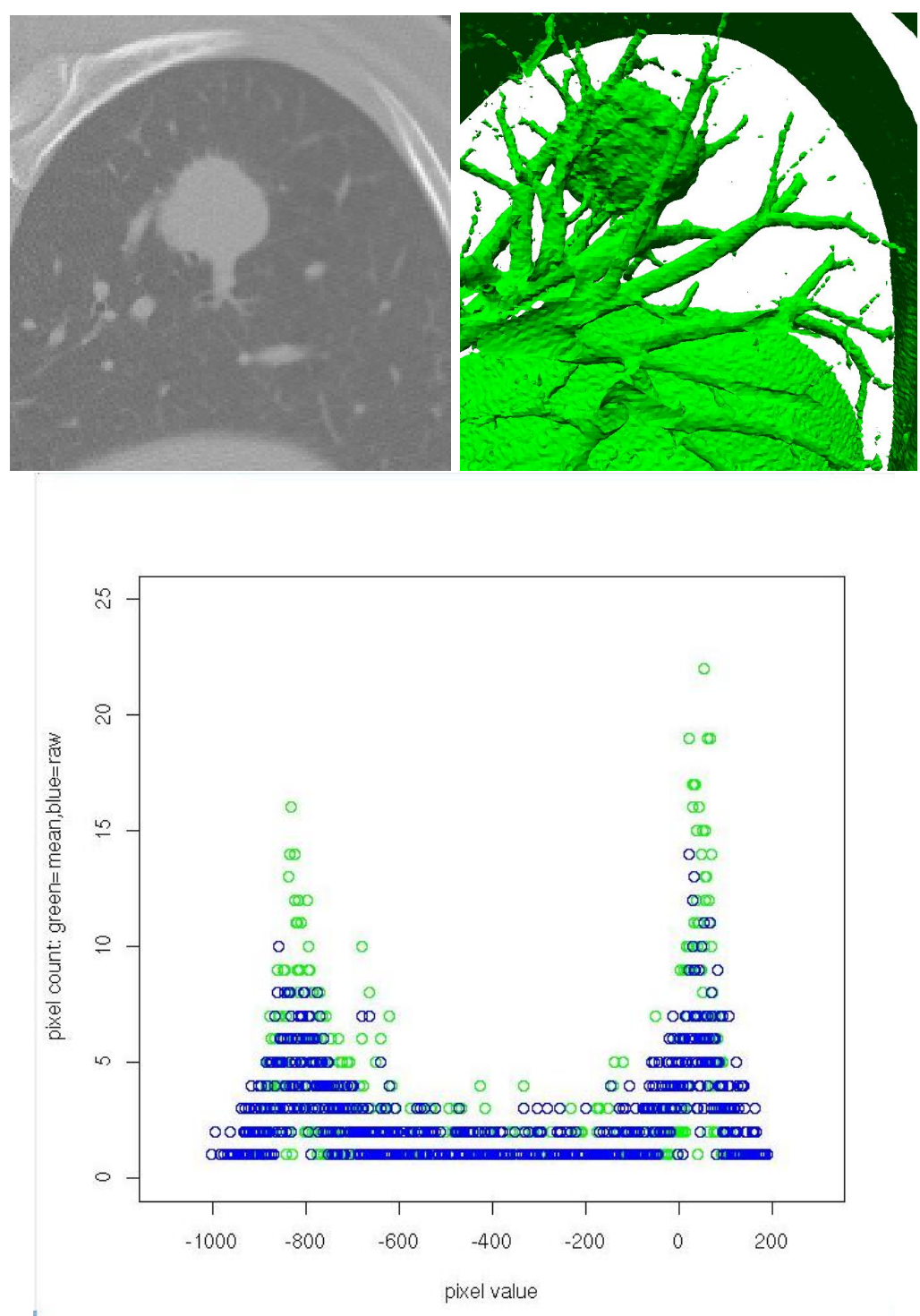

Fig. 1. Section of a slice of CT data containing a tumor; Isosurface at -400 Hounsfield units of 41 slices containing the same tumor; Histogram of pixel intensities in a small grid immediately surrounding the tumor. 
geometric object in the subsections. Using this technique, we are able to get an accurate estimate of the final volume for the combined set of objects. A particular set of geometric objects is assembled in a grid whose grid points contain either a 1.0 for a completely filled voxel, a 0.0 for a completely empty voxel, or a number between 0.0 and 1.0 representing the fraction of the voxel filled by the geometric objects.

As described in a previous paper [9], pixel intensities are then selected for each grid point in the grid of geometric objects. We first approximate the pixel intensity distribution of a clinical tumor in the same data set, using a normal distribution and the mean and standard deviation of the clinical tumor's pixel distribution. Any grid point completely inside of the synthetic tumor is then assigned a random value from this normal distribution.

Pixel intensities for the partially filled voxels are computed based on parameters that we derive from the clinical tumor data, which define the upper and lower boundaries for pixel intensities at the tumor surface. We find the lower boundary for surface pixel intensity from the gradient field of the pixel intensities at the tumor surface. For each intensity at the tumor edge, we find an average value for the magnitude of pixel gradient. $K_{2}$, the lower boundary for surface pixel intensity, is the intensity with the maximum average gradient magnitude at the tumor edge. The upper limit for edge pixels, $K_{1}$, is determined from a ratio based on our previous work. Spheres of different sizes were embedded into clinical CT data such that the pixel intensity gradient at the sphere surface resembled the gradient of clinical tumor data. Pixel intensities at the edge contributed to the overall volumes of the spheres, but were weighted depending upon their intensity. All intensities greater than $K_{1}$ were assumed to be inside the sphere, and because the sphere volumes were known, values of $K_{1}$ were estimated. Over all of the data sets in our previous study, a common ratio emerged from our values of $K_{1}, K_{2}$, and the mean value of the peak of the tumor pixel intensities, $K_{m}$ :

$$
\frac{K_{m}-K_{1}}{K_{m}-K_{2}}=0.46 .
$$

We therefore estimate $K_{1}$ in our current data sets once we know $K_{2}$ and $K_{m}$. Grid values with partial volumes are assigned a pixel intensity between $K_{2}$ and $K_{1}$ according to their partial volume. The new grid is then embedded into the CT data. Figure 2 shows an example of one slice of a sphere as both DICOM data and as colored individual pixels. Table 1 provides data for the range of pixel intensities for the eight synthetic tumor partial volumes, calculated from a clinical tumor found in each of the eight data sets. (See [9] for a lengthier discussion of how and why these parameters are chosen.)

\section{Modeling Clinical Tumor Shapes}

Figure 3 displays the eight geometric objects that were inserted into clinical data. These objects are shown in the figure as polygonal surfaces created from 

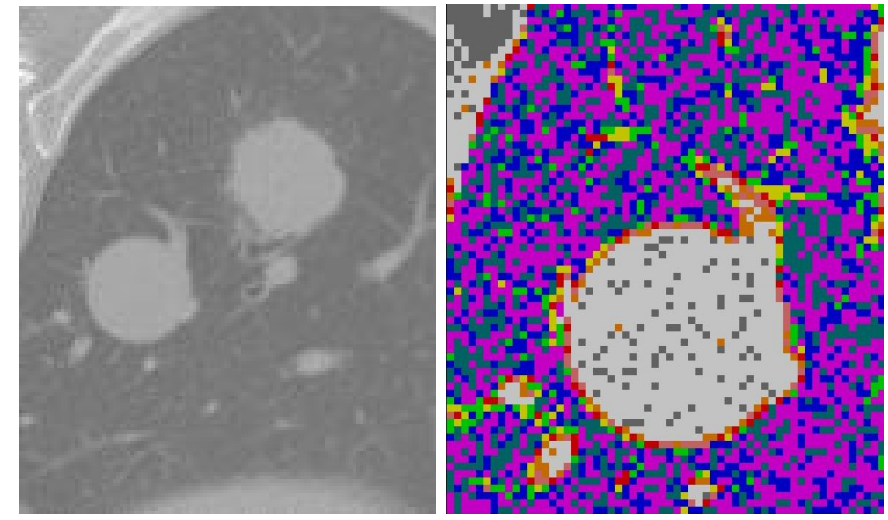

Fig. 2. One slice of a sphere inserted into clinical CT data, DICOM data on the left and pixels individually colored on the right, to differentiate the background, edge, and tumor intensities.

Table 1. Ranges of pixel intensities for partial volumes of synthetic tumors.

\begin{tabular}{ccc} 
Data set & K2 & K1 (Hounsfield units) \\
\hline 1 & -439 & -204 \\
2 & -420 & -194 \\
3 & -413 & -190 \\
4 & -412 & -189 \\
5 & -356 & -167 \\
6 & -388 & -182 \\
7 & -415 & -188 \\
8 & -426 & -197
\end{tabular}


gridded data and represent a range of tumor sizes. Some were embedded in the wall of the lung to simulate more challenging cases; others were kept separate from the wall and are intended to be easier to measure. The clinical tumors that served as a basis for these shapes were selected with the help of a radiologist as representative samples from 26 sets available for this study. Each object in Figure 3 is made up of between four and 13 different geometric shapes chosen so that their cross sections in each CT slice resemble the cross sections of the clinical data. Figures 4-11 display sections of a single slice from each of the eight amended data sets at two different time points. The image data is taken from a single patient at two different times. In several of the figures, the clinical tumors that were used to model the synthetic tumors are seen. The volume change is specified for each set as either a $30 \%$ increase in volume or a $10 \%$ decrease in volume.
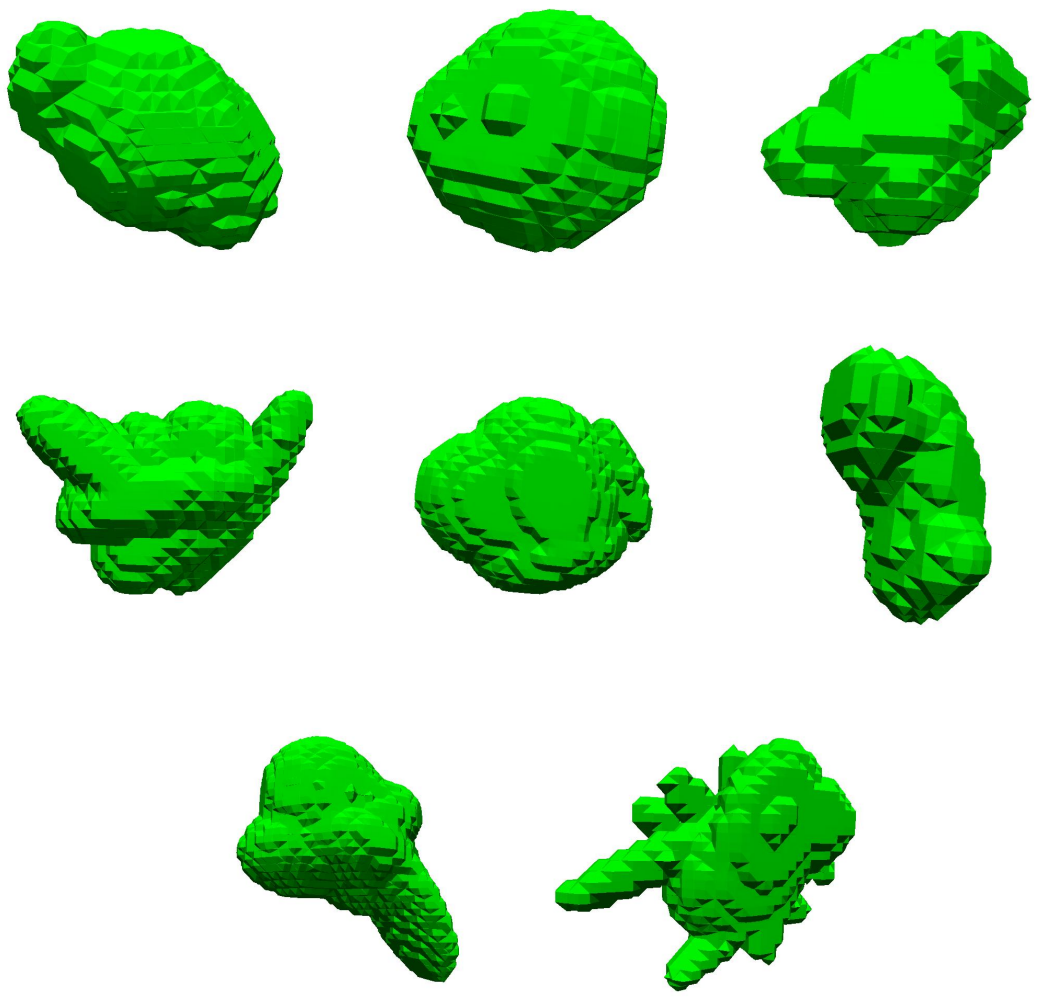

Fig. 3. The eight geometric objects that were inserted into clinical data at the initial time of the test. 

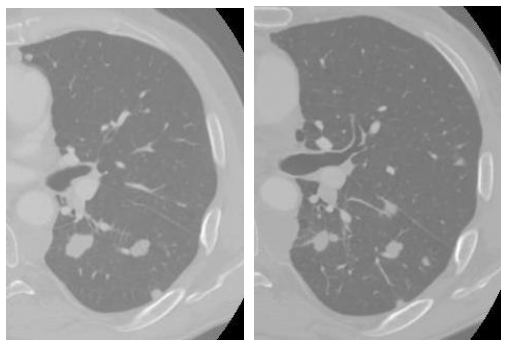

Fig. 4. Object 1 at two time points. The clinical tumor is bottom left; the synthetic tumor to the right of it, modelled after the tumor at time point 2 . Tumor was reduced in size by $10 \%$ for the second time point. The clinical tumor shrank by a larger amount during the corresponding time period.

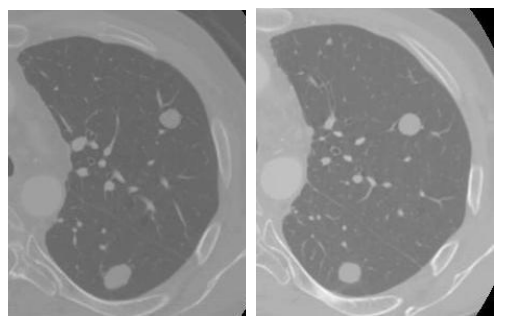

Fig. 5. Object 2 at two time points. The clinical tumor is bottom center; the synthetic tumor is top center, modelled after the tumor at time point 2 . Tumor was increased in size by $30 \%$ for the second time point.

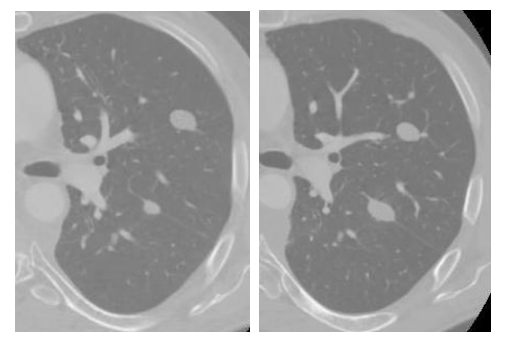

Fig. 6. Object 3 at two time points. The clinical tumor is bottom center; the synthetic tumor is above it, modelled after the tumor at time point 2 . Tumor reduced in size by $10 \%$ for the second time point. 


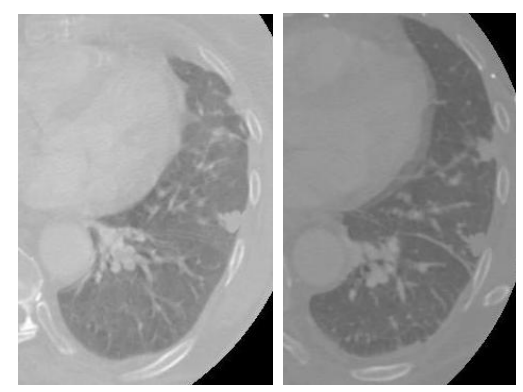

Fig. 7. Object 4 at two time points. The clinical tumor is top right; the synthetic tumor below it, modelled after the tumor at time point 2 . Tumor is increased in size by $30 \%$ for the second time point.
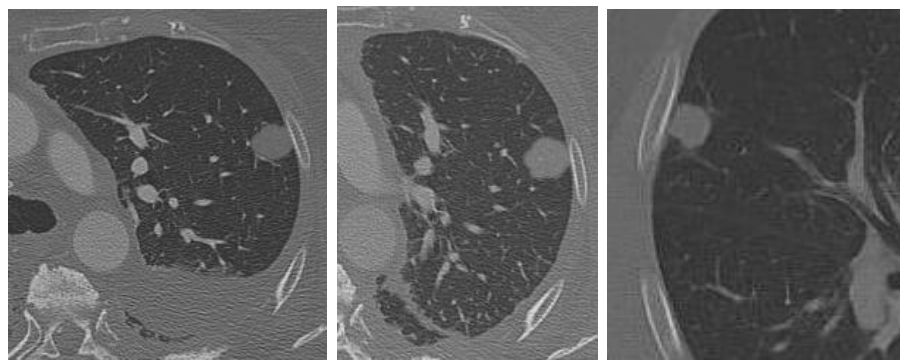

Fig. 8. Object 5 at two time points. The clinical tumor is not in this image; the synthetic tumor is at top right. Tumor is increased in size by $30 \%$ for the second time point. The third image is a section of a slice of the CT data containing the clinical tumor from which the synthetic tumor was derived.
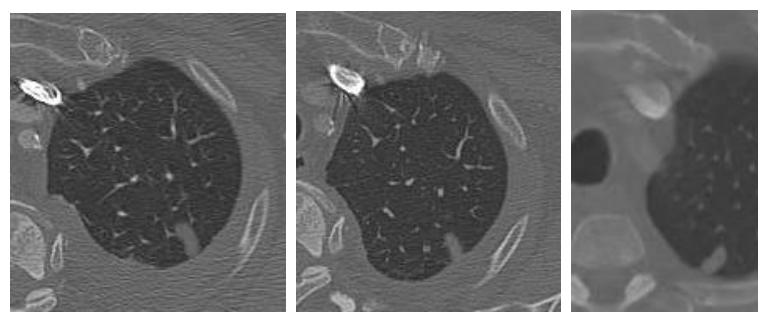

Fig. 9. Object 6 at two time points. The clinical tumor is not in this slice; the synthetic tumor is seen at the bottom of the lung image. Tumor was reduced in size by $10 \%$ for the second time point. The third image is a section of a slice of the data containing the clinical tumor from which the synthetic tumor was derived. 

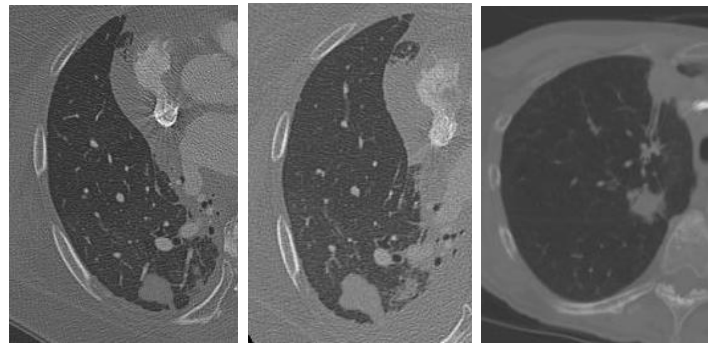

Fig. 10. Object 7 at two time points. The clinical tumor is not in this slice; the synthetic tumor in the lower lung. Tumor was increased in size by $30 \%$ for the second time point. The third image is a section of a slice of the data containing the the clinical tumor from which the synthetic tumor was derived.
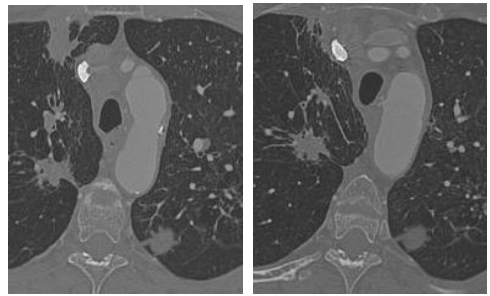

Fig. 11. Object 8 at two time points. The clinical tumor is on the left, the synthetic tumor is bottom right. Tumor was reduced in size by $10 \%$ for the second time point. 
Changes in tumor size over time have traditionally been measured using the Response Evaluation Criteria in Solid Tumors (RECIST). One recent study [11] with phantom tumors has shown that size measurements are dramatically improved when using volumetric measurements instead of one-dimensional RECIST measurements. However, for small tumors, volume measurements often are dependent upon determining exact surface locations and are heavily influenced by the difficulties of measuring partial volumes. This is illustrated in Table 2 , which shows the tumor volumes associated with the eight tumor shapes described here along with the fraction of partially filled voxels for each grid. These partially filled voxels are at risk for error in volume calculations. The fractions vary between 0.247 and 0.410 ; i.e., in our sample, approximately one quarter to one half of the voxels representing tumors are partially filled. Volumetric methods that do not accurately account for partial volume data will lead to errors of this extent. The fractions are high, either because the tumors are small and the surface area to volume ratios are high, or because the tumors, although larger, are less round, with more surface area in extended appendages. This underscores the need for accurate methods to determine tumor boundaries in CT data. We have suggested such a method in [12], which assigns weighted volumes to voxels that are not completely filled. Further work investigating changes in tumor shapes with time are needed to determine if the data sets used here are truly representative.

Table 2. Volumes and partial volumes for the eight objects.

\begin{tabular}{ccc} 
Data set & volume $\left(\mathrm{mm}^{3}\right)$ & fraction of partial volume voxels \\
\hline 1 & 27266.40 & 0.304 \\
2 & 20800.67 & 0.323 \\
3 & 10094.76 & 0.384 \\
4 & 91280.65 & 0.336 \\
5 & 18832.44 & 0.299 \\
6 & 6136.94 & 0.410 \\
7 & 57754.50 & 0.247 \\
8 & 15158.10 & 0.377
\end{tabular}

\section{Conclusions}

Reference CT data sets that contain time-dependent lung tumor size change data are needed in order to compare volumetric methods for measuring lung tumor growth. Phantom lung tumor data can supply time dependent information, but not necessarily in the clinical environment of common lung tumors. These data also often have the disadvantage of not replicating the complications associated with tumor size measurements, such as highly concentrated vasculature or air 
passageways, or growth out of the pleural lining. Creating synthetic data in realistic environments is one way of mitigating these disadvantages. We have shown a selection of clinical tumors from available data that we believe exhibits representative shapes and placements. We describe the insertion of these synthetic shapes into clinical CT data. These representative synthetic tumors can then be used as ground truth data for comparison of algorithms that calculate tumor volumes. We show from an analysis of the gridded data representing all eight of our tumor shapes that a large fraction of voxels are only partially filled. Future work is needed to further investigate tumor shapes and their changes over time to see if our small study is representative of lung tumor data in general.

\section{Acknowledgements}

\section{References}

1. Kostis, W.J., Reeves, A.P., Yankelevitz, D.F., Henschke, C.I.: Three-Dimensional Segmentation and Growth-Rate Estimation of Small Pulmonary Nodules in Helical CT Images. IEEE Trans. on Medical Imaging, 22(10), (October 2003)

2. Anthony P. Reeves, A. B. Chan, David F. Yankelevitz, Claudia I. Henschke, B. Kressler, William J. Kostis: On measuring the change in size of pulmonary nodules. IEEE Trans. Med. Imaging, 25(4), 435-450 (2006)

3. P. Mendonca. R. Bhotika, S. Sirohey, W. Turner, J. Miller, R.S. Avila: Modelbased Analysis of Local Shape for Lesion Detection in CT Lung Images. Proceedings of the International Conference on Medical Image Computing and ComputerAssisted Intervention (MICCAI 2005), (October 2005)

4. McCulloch,C.C., Kaucic, R.A., Mendonca, P.R., Walter, D.J., Avila, R.S.: Modelbased Detection of Lung Nodules in Computed Tomography Exams. Academic Radiology, (March 2004)

5. Preim, B., Bartz, D.: Image Analysis for Medical Visualization. Visualization in Medicine, 83-131, (2007)

6. Preim,B., Bartz, D.: Exploration of Dynamic Medical Volume Data. Visualization in Medicine, 83-131, (2007)

7. Das, M., Ley-Zaporozhan, J., Gietema, H.A., Czech, A., Nuhlenbruch, G., Mahnken, A.H., Katoh, M., Bakai, A., Salganicoff, M., Diederich, S., Prokop, M., Kauczor, H., Gunther, R.W., Wildberger, J.E.: Accuracy of automated volumetry of pulmonary nodules across different mutlislice CT scanners. Eur Radiol, 17, 1979-1984, (2007)

8. Ko, J.P., Rusinek, H.,Jacobs, E.L., Babb, J.S., Betke, M., McGuinness, G., Naidich, D.P.: Small Pulmonary Nodules: Volume Measurent at Chest CTPhantom Study. Radiology, 228:864-70, (2003)

9. Peskin, A.P., Kafadar, K., Dima, A., Bernal, J., Gilsinn, D.: Synthetic Lung Tumor Data Sets for Comparison of Volumetric Algorithms. The 2009 International Conference on Image Processing, Computer Vision, and Pattern Recognition. 4347 (July 2009)

10. NCI Reference Image Database to Evaluate Response (RIDER) database. https://wiki.nci.nih.gov/display/CIP/RIDER

11. Levine,Z.H., Borchardt,B.R., Brandenburg,N.J. Clark,C.W., Muralikrishnan,B., Shakarji,C.M., Chen,J.J., Siege:,E.L. RECIST versus volume measurement in 
medical CT using ellipsoids of known size. Optics Express, 18(8), 8151-8159 (April 12, 2010)

12. Peskin, A.P., Kafadar, K., Santos,A.M., Haemer,G.G.: Robust Volume Calculations of Tumors of Various Sizes. The 2009 International Conference on Image Processing, Computer Vision, and Pattern Recognition. (July 2009) 